\title{
An Improved Technique to Enhance High-Iron Diamine Reactive Sites with Thiocarbohydrazide-Silver Proteinate
}

\author{
by \\ Francis R. DENYS 1 , Minoru TAKAGI ${ }^{2}$ and Richard T. PARMLEY ${ }^{3}$
}

SPICER's high-iron diamine (HID) method (SPICER 1965) has been applied ultrastructurally to demonstrate sulfated glycoconjugates in various tissues (SPICER et al., 1978, SANnes et al., 1979, TAKAgi et al., 1981 and TAKAGI et al., 1982). SANNES et al. (1979) successfully devised a method to enhance HID reactive sites with thiocarbohydrazide-silver proteinate (TCH-SP). The specificity of the HIDTCH-SP method for sulfate has been corroborated by ultrastructural radioautographic studies localizing ${ }^{35} \mathrm{SO}_{4}$ (TAKAGI et al., 1981), enzyme digestion studies demonstrating lability of the HID stained material (TAKAGI ET ALal., 1982) and comparison to immunocytochemical stdiues (TAKAGI et al., 1982) utilizing a rabbit antiserum specific for chondroitin sulfate. The present report concerns the description of the TCH-SP method to enhance HID staining using a new technique which involves the immersion of specimen grids in stain solutions so as to minimize background staining and provide uniform staining.

Biological tissues, which were obtained from various animals, were fixed in $2.7-3 \%$ glutaraldehyde in $0.1 \mathrm{M}$ cacodylate buffer $\left(\mathrm{pH} 7.35\right.$ ) for $1-3$ hours at $4^{\circ} \mathrm{C}$. After fixation, ethylene-diamine-tetraacetic acid (EDTA) demineralization was performed for highly calcified tissues. After rinsing several times in $0.1 \mathrm{M}$ cacodylate buffer $(\mathrm{pH} 7.35)$ containing 7\% sucrose, the specimens were stained for 18 hours at $22^{\circ} \mathrm{C}$ in the HID staining solution ( $\left.\mathrm{pH} 1.4\right)$ prepared as previously described (SPICER, 1965). Control specimens (for evaluation of intrinsic density) were also processed as previously described. The specimens were then postfixed in $1 \%$ or $2 \% \mathrm{OsO}_{4}$, routinely dehydrated, and embedded in Spurr low viscosity resin. Recently $1 \% \mathrm{OsO}_{4}$ has been used in the postosmication step in our laboratories, since $2 \% \mathrm{OsO}_{4}$ produced more $\mathrm{TCH}-\mathrm{SP}$ background staining in osmiophilic sites. The postosmication step was omitted for some specimens.

The sections were collected on stainless steel grids. As the grids had to go into two staining solutions and were rinsed a total of 10 times, a method was used to facilitate the transfer of the grids from one solution into another. Holding the grid with fine forceps, a hole was made into the grid with a $25 \mathrm{~g} 5 / 8$ inch hypodermic needle (Fig. 1). Nichrome wire (30 gau., Ted Pella, Inc., Tustin, CA, U.S.A.) was threaded through the hole, the ends held together and wrapped around a piece of

\footnotetext{
${ }^{1-3}$ From the Institute of Dental Research, the University of Alabama in Birmingham, Birmingham, Alabama 35294; and ${ }^{2}$ the Department of Anatomy, Nihon University School of Dentistry, 1-8-13, Kanda-Surugadai, Chiyoda-ku, Tokyo 101, Japan.
} 
applicator stick. When many grids were stained, a piece of tape was attached at one end of the applicator stick and numbered (Fig. 2). The staining solutions and rinsing water were poured into clean shell vials $(15 \times 45 \mathrm{~mm})$ (Fisher Scientific Co., Fair Lawn, NJ, U.S.A.). The grids were hung in the solutions and easily transferred from one vial to another (Fig. 3).

For the best staining results, TCH and SP solutions should be made fresh and staining must be performed within a week after making these solutions. Sections

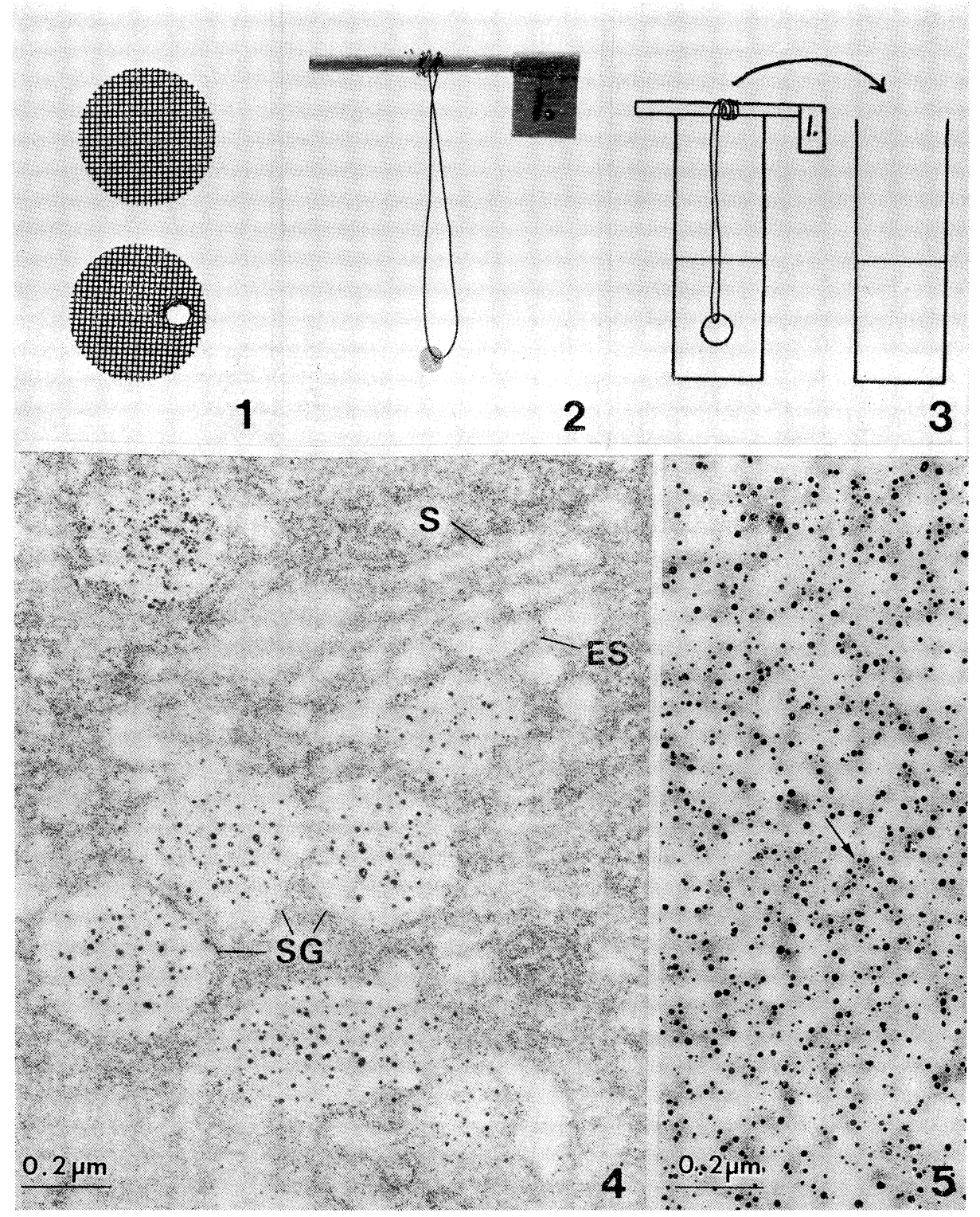


were treated for 40 minutes with $2 \%$ thiocarbohydrazide (Eastman Kodak Co., Rochester, NY, U.S.A.) in $20 \%$ acetic acid (Fig. 3). This staining solution was filtered with Whatman filter $\# 2$ before use. After rinsing in $10 \%$ acetic acid and four times in distilled water, they were treated for 30 minutes with $1 \%$ silver proteinate (strong silver protein, Roboz Surgical Instrument Co., Inc., Washington, D.C., U.S.A.) in distilled water in the dark. A safety light (Kodak filter OA) could be used in the darkroom. SP background staining was eliminated by filtering (Whatman filter \#2) the SP solution twice before use. Subsequently, the specimens were rinsed six times in distilled water and examined with a Philips 300 electron microscope.

TCH-SP staining of osmicated and unosmicated specimens significantly enhanced the HID reactive foci in chondrocytes (Fig. 4) and the extracellular cartilage matrix (Fig. 5). TCH appeared to bind HID reactive sites (presumably aldehydes in the oxidized diamine) and then reduce SP to form electron dense stain deposits. The size of the stain deposits may represent variability in the amount of sulfate in the reactive site (TAKAGI et al., 1982). $\mathrm{OsO}_{4}$ postfixation did not influence the size of the TCH-SP stain deposits, but occasionally resulted in an increase in fine background stain deposits.

In summary, this application technique allows ultrastructural localization of HID reactive sulfated glycoconjugates in various tissues without background staining and contamination.

\section{Acknowledgments}

This work was supported in part by Grant DE-02670 from the National Institutes of Health. The authors wish to thank Ms. Barbara A. Woolley for her secretarial assistance.

\section{Abstract}

An improved method is described for staining sulfated glycoconjugates with

$\leftarrow$ Fig. 1 A stainless steel grid before and after a hole is made with a $25 \mathrm{~g} 5 / 8$ inch hypodermic needle.

Fig. 2 A nichrome wire is threaded through the hole and attached to a piece of applicator stick. When several grids are stained simultaneously, a number is attached for identification.

Fig. 3 The grid is hung in the solution during staining and rinsing, and can be easily transferred from one vial to another.

Fig. 4 In rat epiphyseal cartilage, HID-TCH-SP stain deposits can be seen in chondrocyte Golgi expanded saccules (ES), Golgi derived vacuoles and secretory granules (SG) at various maturational stages, but are not present in Golgi saccules (S). Specimen postfixed with osmium tetroxide after HID stain. $\times 62,500$.

Fig. 5 HID-TCH-SP stain deposits localize material presumed to be proteoglycan monomer(s) in the cartilage matrix granule (arrow). Note the presence of two populations of stain deposits, one averaging $7 \mathrm{~nm}$ in diameter, the other $11 \mathrm{~nm}$ in diameter. Many of the small stain deposits persist after testicular hyaluronidase digestion and are presumed to be keratan sulfate. Unosmicated specimen. $\times 62,500$. 
high-iron diamine (Spicer, 1965)-thiocarbohydrazide-silver proteinate (Sannes et al., 1979). This method results in uniform staining of thin sections with minimal or no background.

\section{References}

[1] Sannes, P. L., Spicer, S. S. and Katsuyama, T. 1979. Ultrastructural localization of sulfated complex carbohydrates with a modified iron diamine procedure. J. Histochem. Cytochem., 27, 1108-1111.

[2] SPICER, S.S. 1965. Diamine methods for differentiating mucosubstances histochemically. $J$. Histochem. Cytochem., 13, 211-234.

[3] Spicer, S. S., Hardin, J. H. and SetSer, M. E. 1978. Ultrastructural visualization of sulfated complex carbohydrates in blood and epithelial cells with the high-iron diamine procedure. Histochem. J., 10, 435-452.

[4] Takagi, M., Parmley, R. T. and Denys, F. R. 1981. Ultrastructural cytochemistry and radioautography of complex carbohydrates in secretory granules of epiphyseal chondrocytes. Lab. Invest., 44, 116-126.

[5] Takagi, M., Parmley, R. T., Toda, Y. and Austin, R. L. 1982. Ultrastructural cytochemistry and immunocytochemistry of sulfated glycosaminoglycans in epiphyseal cartilage. J. Histochem. Cytochem., 30, 1179-1185. 